\title{
Research on P.E. at school based on complexity theory of visual threshold
}

\author{
Lu Yun Fei \\ Wuhan University of Science and Technology \\ Wuhan, Hubei 430070, China
}

\begin{abstract}
School physical education and the conventional methods and form has been hard to master many complex problems facing the modern school physical education. School physical education is mainly based on the complexity science is different from the previous "linear" and "reductionism" way of thinking, "to the non-linear thinking, thinking, thinking, thinking the relationship between operation mode of school physical education. This paper mainly describes the physical education as a complex system contains, complexity of education object, education structure, education method, education environment. At the same time, points out that school physical education should go beyond the reduction thinking, realize the education as a whole, to prevent the dependence of physical education, emphasis on physical education consciousness of reflection, to break the conservative thinking mode of static, physical education, innovation, simplistic tendency to reverse the education method, attach importance to comprehensive education method.
\end{abstract}

Keywords- complexity; school sports ; education

\section{INTRODUCTION}

World view in classical science believes that the world is ordered in nature, disorder belongs to phenomena of matters only, the target of scientific knowledge is to find out and master the regularity of nature of matters through troublous phenomena. P.E. world is a world in which order and disorder, certainty and uncertainty, simple and complex blend with each other and complicated procedure in which various influencing factors disintegrate and reorganize continuously, harmoniousness and noises interweave repeatedly. Once P.E. at school in modern times falls into the thinking method of reductionism, it would confront with a series of contradictions and conflicts due to connatural defects of the method inevitably, which leads to existential crisis of failures of educational significance in P.E. at school. The real world is not separated, in pieces and carsal, as Pascal said, anything is the reason and the result and has indirect and direct connections, all the matters are connected naturally. The reality requires people to seize general and basic issue to consider through complicated ways of thinking.

\section{COMPLEXITY IN P.E. AT SCHOOL}

\section{A. Complexity in educational objects}

Complexity in educational objects leads to complexity in education directly. From ancient times to present, people have revealed on the issue of human nature at different sides in different degrees. In modern times, people have recognized that humans are not only the substance on individual layer but on social and cultural ones. Marx pointed out that human being is the sum of all social relations. Easy language has fully shown the human complexity. Cahir has revealed human complexity from the literary respective, " human culture as a whole can be regarded as a procedure of continuous self-liberation." Morelan believes that "one funeral bell has rung on closed, fragmentary as well as simplified theory for human beings and an open, multifaceted as well as complicated theoretical era has begun." [3]

\section{B. Complexity in educational methods}

Complicated casual linkages existed among various influencing factors in the educational world. Mike Fran pointed out in summarizing the complicated revolutionary patterns of education that "revolution is a journey but not a blueprint", because "revolution is non-linear which is full of uncertainties and excitements, sometimes violates common sense", furthermore, we "could not decide what is important by force because the more complicated the revolution is, the less you could compel it to do." [5] P.E. at school has its complex property and the complex thinking stresses that the development of matters is non-linear meanwhile stresses that there is universal connection among different matters, this connection is particularly obvious in the procedure of P.E. at school. With the booming of P.E. business, people have fully recognized the more complex subject interest and mission of P.E. at school as a special kind of social activity and must required specificity and pluralism in educational methods.

\section{Complexity in educational environment}

Complexity in microenvironment of P.E. at school is mainly determined by internal structure in it. Firstly, different targets of P.E. at school determine its different spaces, different projects require different athletic fields meanwhile require safety in different projects teaching aiming at dangerous kinds, complexity of educational environment on this degree mainly depends on teaching of different projects and rational allocation of grounds. In addition, cultural environment of P.E., this complexity mainly depends on interest degrees of students on different projects, how to deal with the educational environment caused by differences among different projects in microenvironment has become one of the important reasons in complexity of teaching environment. 


\section{Complexity in evaluation methods}

Evaluation of P.E. at school is largely different from other subjects such as math exam, Chinese exam as well as English exam, this exam mainly focuses on exam results of students, that is, focuses on qualitative evaluation. Due to specificity in P.E. at school, evaluation of P.E. at school has its unique evaluation ideas, not only focus on evaluation results but on evaluation procedure. Evaluation methods of P.E. at school pay attention to these relations meanwhile stands on the final requirement of expecting individual development of students, P.E. teachers shall select evaluation methods properly in comprehensive evaluation, which could both correct learning attitude of students with good results and strengthen learning confidence in students with "poor results". Here we could see that due to specificity in P.E. at school in itself and complexity of educational objects, evaluation ideas cannot be in accordance with summative assessment with simple way of thinking, this is the key where complexity of P.E. evaluation lies.

\section{E. Complexity in course curriculum in P.E. at school}

P.E. curriculum is the core of P.E. at school and media and carrier of educational activities at school. P.E. curriculum is static in itself but its procedure of significance production is dynamic. Setting of P.E. curriculum at school shall be constrained by social environment to some extent, meanwhile consider main development of students, in drifting away between the two, it has to continue to absorb different educational ideas to expect balance between the two, which could not only develop students capacities to let P.E. return to "education" truly but consider the social requirements to provide motive force for development. Under comprehensive influences of various factors, how to rationalize setting of P.E. curriculum at school and how to spur it to develop educational value in maximum, applying complexity thinking to weigh relations among different factors and getting rid of the control of traditional linear thinking is one of the important features in its complexity.

\section{P.E. AT SCHOOL FORWARDING TO COMPLEXITY THINKING IN OUR COUNTRY}

\section{A. Goes beyond thinking in reduction and realize integrated education}

From the perspective of complexity thinking, interaction among various parts of P.E. at school is not in simple plus relationship, the causality among them is not unidirectional chain but complex causality net. P.E. target at school determines development direction of P.E. at school, P.E. curriculum is the core of P.E. at school, educational target in its kind would have corresponding curriculum and corresponding teaching methods. Interaction among various parts in P.E. at school leads to uncertainty of the system as a whole even unexpected overall characters. Confronted with complexity in P.E., any subject in education only solves one local problem, only intensive research on many subjects could we solve the problem as a whole. Therefore, in conducting P.E. at school, we not only consider to realize the main target but consider influences of periodic target on other factors of the system during different periods as far as possible and overcome narrow boundary consciousness of subjects, absorbing advanced teaching idea positively and abandoning easy reduction thinking, forming diversified and integrated thinking pattern, regarding problems occurred in P.E. as complete, complicated as well as irreversible substances.

\section{B. Prevent educational anaclisis and stress educational awareness of reflection}

Anaclisis of P.E. at school is mainly because the theoretical research in P.E. at school is late in start, although the scientific frame of P.E. in our country has basically formed and many treaties with Chinese characteristics have appeared, P.E. theories in our country still have strong anaclisis from the perspective of theory construction mode, this has fully demonstrated that the result is to take and transplant certain achievements in foreign P.E. without differentiation and analysis due to lack of intensive analysis combined with Chinese realities, it is always difficult to learn its essence and deviations would occur in theory and misleads in practice.

\section{Breaks up static and conservative way of thinking and innovates educational methods}

P.E. at school is transforming from "training" to teaching method of strengthening physics, development innovation from happy P.E. to lifelong P.E., from physical education to quality education at present. Many teachers are still inheriting the traditional teaching ideas in teaching practice and not conducting teaching creatively. For instance, the content of P.E. in our country has failed to lead P.E. in depth and stopped on phenomenon and substance. Teaching some motor skills directly cannot satisfy requirements of students on recognition on P.E. Because teaching "forward roll" and "shot put" cannot reach the final target of P.E. but reach to grasp the essence of P.E. until the educational target through teaching of "forward roll" and "shot put". Furthermore, P.E. teaching and research groups are uneven in level, many schools do not have teaching and research group and more schools are not clear of responsibilities of teaching and research group. One of important responsibilities of P.E. teaching and research group is to organize P.E. teachers to conduct irregular theory study, learn up-to-date knowledge in education, understand new development trend in P.E. at school and discuss actively how to innovate teaching methods better. This has illustrated that static and conservative way of thinking is difficult to adapt to requirements of P.E. at school in modern times.

\section{Turn around simplification tendency of educational methods and attach importance to integration of educational methods}

I believe that P.E. methods at school in our country at present show simplified tendency mainly because firstly, educational level or knowledge of educators is limited; secondly, educators cannot hold subject characteristics of P.E. at school clearly; thirdly, educators did not take changes 
in P.E. environment at school into account. As far as the educational environment is concerned, it mainly divides into internal factors and external factors. Education in internal factors also shows multilevel and comprehensiveness. It covers subjective factor and objective factor of education. External environment shows the complexity which mainly includes natural environment, social environment, economic environment, cultural environment as well as population environment and so on. Because P.E. at school involves many educational subjects, different subjects have different teaching methods, in which the subjective factor, that is the teacher, and the objective factor, that is the student are the important indexes to determine which teaching method to adopt, however, due to similarities among many projects such as tennis and badminton, sports dance and aerobics, how to address this similarity has become the key in selecting teaching methods, of course, for those students with different characters, teaching students in accordance with their aptitude is also the index in selecting educational method.

Up to now, most of P.E. theory researches are still on reductionism generally, focusing on list of factors and analysis of factors but ignoring interaction, mutual locking, dynamic conversion as well as collective generation of many external factors of P.E., did not regard P.E. activities as a complex activity as a whole to recognize and research. Under this thinking mode, P.E. at school would simplify in teaching methods inevitably. All in all, due to inherent complexity in sports system, methods that research on issue of P.E. complex system will certainly go forward to comprehensiveness.

\section{CONCLUSION}

Marxism theory believes that anything is interconnected, this connection may be direct or indirect, complexity thinking stresses non-linear connection among matters, main subject and object in P.E. at school are "humans", while this connection among people seems direct and linear seemingly; in fact, there are full of numerous subtle changes under this surface phenomenon. According to the analysis of contradiction theory in Marxism, the main contradiction in P.E. at school is the contradiction between the teacher and the students, the main facet of the contradiction is the contradiction between all-around development and lopsided development. How to deal with the main contradiction between teacher and the students and how to promote allaround development of students have become the significant factor in solving the problem with complexity thinking in P.E. at school. Of course, research on the issue of P.E. at school under complexity theory is also a breakthrough under easy thinking theory. Regarding various kinds of problems occurred in P.E. at school as a complex and comprehensive problem, positively absorbing advanced educational ideas to conduct analysis on it, it not only could obtain unexpected effect but could expand research field of P.E. at school which provides more solid theoretical support for science of P.E. at school.

\section{Reference}

[1] France] Edgar Moran Complexity thinking: conscious science [M]. Translated by Chen Yi Zhuang . Beijing: Beijing University Press, 2001.06: 211

[2] [France] Edgar Moran Introduction of complexity thinking [M] Translated by Chen Yi Zhuang. Shanghai: East China Normal University Publishing House, 2001.06: 35

[3] [France] Edgar Moran The lost pattern: Human nature research [M] Beijing: Beijing University Press, 1997.07: 173

[4] G Moorman Development trend in education[A]. Translated by Zhao Wei Xian. Chief editor Qu Bao kui. Collection of educationEducation and pedagogy[M]. Beijing: People's Education Press, 1993.04: 89 